\title{
A NOTE ON FINITE GROUP STRUCTURE INFLUENCED BY SECOND AND THIRD MAXIMAL SUBGROUPS
}

\author{
N.P. MUKHERJEE and R. KHAZAL \\ Department of Mathematics \\ Kuwait University \\ P.D. BoX 5969, 13060, Kuwait
}

(Received July 20, 1988)

ABSTRACT. The structure of a finite group having specified number of second and third maximal subgroups has been investigated in the paper.

KEY WORDS AND PHRASES. Maximal subgroup, solvable, supersolvable, nilpotent. 1980 AMS SUBJECT CLASSIFICATION CODE. 20D10.

\section{INTRODUCTION.}

It is easy to see that a group $G$ with exactly one maximal subgroup $M$ is cyclic since the Frattini subgroup $\Phi(G)$ coincides with $M$. In this note the structure of groups having one/two/three second maximal subgroup and groups having one/two-third maximal subgroups are investigated. All groups considered here are finite and the notations used are all standard.

For the sake of completeness we mention below the following theorem or p-groups [Theorem 7.6, p. 304] in Huppert [3] which will be used.

THEOREM H. Let $G$ be a p-group and suppose all abelian normal subgroups of $G$ are cyclic. Then, (a) $G$ is cyclic if $p>2$ (b) if $p=2$, $G$ has a cyclic normal subgroup of index 2 .

We will first characterize groups having the desired number of second maximal subgroups. The following lemmas will be required.

\section{GENERAL RESULTS.}

LEMMA 2.1. A p-group $G$ which has exactly one nontrivial second maximal subgroup is cyclic if $p>2$ and has a cyclic normal subgroup of index 2 if $p=2$. PROOF. Evidently $|G|>p^{2}$. Let $|G|=p^{n}, n>2$ and $M$ be a maximal subgroup $G$. Then $M \unlhd G,|M|=p^{n-1}>p$ and therefore the given second maximal subgroup $M_{0} \underset{q}{M} M_{0}$

being the only maximal subgroup of $M$, it follows that $M$ is cyclic and therefore each abelian normal subgroup of $G$ is cyclic. From Theorem $H$ it now follows that $G$ is of the desired type. \footnotetext{
or $p^{2}$ or $q t, p, q, t$ are different primes.
} 
PROOF. If $G$ is a $p$-group and $G$ has no second maximal sulgruup then evidently $|G|=p$ or $p^{2}$. Now suppose $G$ is not a $p$-group, then every maximal subgroup is of prime order and therefore the sylow subgroups of $G$ are cyclic and are of prime orders. Consequently $G$ is supersolvable and $G$ has a Sylow basis. If $|G|$ is divisible by more than two primes then clearly $G$ will have a second maximal subgruup and therefore the order of $G$ must be $q t$, for some primes $q$ and $t$.

THEOREM 2.1. A group $G$ having exactly one second maximal subgroup is a p-group. For $p>2, G$ is cyclic and for $p=2$, G has a cyclic subgroup of index 2 .

PROOF. It suffices to show that $G$ must be a p-group if it has one second maximal subgroup. Lemma 2.1 will then guarantee the structure of $\mathrm{G}$ as claimed.

Suppose $G$ is not a p-group and without loss of generality we may assume $G$ to be a counter example of least possible order. Then every maximal subgroup of $G$ is either of prime order or else contains the given second maximal subgroup $M_{0}$ of $G$. In the latter case, $M$ is clearly cyclic of prime power order. Thus every maximal subgroup of $G$ is cyclic and therfore every sylow subgroup of $G$ is cyclic and $G$ is supersolvable. Hence $G=P K, P \equiv G, P \cap K=1$ where $P$ is the Sylow subgroup corresponding to the largest prime divisor $p$ of $|G|$ and $K$ is a p-complement.

Let $|K| \neq 1$. Now $K$ has at most one second maximal subgroup. If $K_{1}$ and $K_{2}$ are two second maximal subgroups of $K$ then $P K_{1}$ and $P K_{2}$ are two different second maximal subgroups of $G$, a contradiction. (If $P K_{1}=P K_{2}$ then $K_{1} \neq K_{2}$ implies that some $u \in K_{1}, u \notin K_{2}$ and it follows that $u=x v, x \varepsilon P, v \varepsilon K_{2}, x \neq e$. Then $u v^{-1}=x$. But $u^{-1} \varepsilon K$, and is therefore a $p^{\prime}$ element. Hence $\left.P K_{1} \% P K_{2}\right)$. If $K$ has exactly one second maximal subgroup then $K$ is a $q$-group for some prime $q$ since $G$ is the minimal counter example and if $K$ has no second maximal subgroup then we need consider $|K|=r^{2}$ or st, $r, s, t$, are different primes. We first consider this latter case.

CASE I. $|K|=r^{2}$. Consider the subgroup $p\langle u\rangle,|\langle u\rangle|=r$. $M=P\langle u\rangle$ is a maximal subgroup $G$ and $P$ is the second maximal subgroup. Hence $\phi(M)=P$ and $P Y|M|$, a contradiction. Thus $|\mathrm{K}| \neq \mathrm{r}^{2}$.

CASE II. $|K|=s t$. Let $G=P S T$, where $S$ and $T$ are Sylow subgroups of $G$ of orders $s$ and $t$ respectively. $M_{1}=P S$ is a maximal subgroup and $P$ is the second maximal subgroup of $G$. Hence $\phi\left(M_{1}\right)=P$ and $P Y\left|M_{1}\right|$, a contradiction. Therefore $|K| \neq s t$.

We are thus left to consider the case when $K$ is a q-group. Distinguish two cases: $\operatorname{CASE} A \cdot|K|=q$. Observe that $|P| \neq p$ as otherwise $|G|=p q$ and $G$ will have no second maximal subgroup. We may therefore assume $|P|=p^{n}, n>2$. Let $P_{1}$ be maximal in $P$ so that $\left|P_{1}\right|=p^{n-1}$. Note $P_{1} \unlhd G$ since $P$ is cyclic and $P \unlhd G$. Consider $P_{1} K$, a maximal subgroup of $G$. If $P_{2}$ is maximal in $P_{1}$ then $P_{2} \unlhd G$ and $P_{2} K$ and $P_{1}$ are two second maximal subgroups of $G$, contradicting the existence of only one.

CASE B. $|K|=q^{m}, m>2$. First suppose $|P|=p$ and let $K_{1}<k$. Then $\left|K_{1}\right|=q^{m-1}$ and consider $P K_{1}$. If $K_{2}<$. $K_{1}$ then $P K_{2}$ and $K_{1}$ are two second maximal subgroups of $G$ which contradicts again the existence of only one. Now suppose $|P|=p^{n}, n>2$ and let $p_{1}<\cdot p$ and $K_{1}<K_{\text {. }}$ Both $P_{1} K$ and $P K_{1}$ are maximal subgroups of G. If $P_{2}<. P_{1}$ and $K_{2}<K_{1}$ then $P_{2} K$ and $P_{2}$ are second maximal subgroups of $G$ which is a contradiction and so $|k|$ must be 1 . Hence $G$ is a p-group. Thus there is no minimal counter example and the theorem follows. 
$\therefore$ nuw investigate the structure of groups with two second maximal subgroups. The following lemma is necessary.

LEMMA 2.3. A group $G$ with exactly two maximal subgroups is necessarily cyclic and the order of the group is divisible by two distinct primes.

PROOF. If either of the given maximal subgroups is not normal then it being its own normalizer is of index 2 which however forces it to be normal. Thus $G$ is nilpotent and we claim that $G$ cannot be a p-group.

Suppose the set $S=\{X \mid X$ is a $\mathrm{p}$-group and $X$ has exactly two maximal subgroups $\}$ $\neq \phi_{0}$ If $S_{1}=\{Y \mid Y \in S$ and $Y$ is not cyclic $\} \neq \phi$ then $T_{0}$ is an element of $S_{1}$ of least possible order.

Suppose $M$ and $M^{*}$ are the two maximal subgroups of $Y_{0}$ and $\left|Y_{0}\right|=p^{n}$. Distinguish two cases: Case $I$. $M \cap M *=\langle e\rangle$. Since $M$ and $M^{*}$ are normal in $Y_{0}, Y_{0}=M^{*}$ and $\left|Y_{0}\right|=|M| \cdot\left|M^{*}\right|$. Thus $p^{n}=p^{n-1} \cdot p^{n-1}$ and $p^{n}=p^{2}$. Therefore $Y_{0}$ is elementary abelian and $Y_{0}$ is not cyclic. If $a$ and $b$ are elements of $Y_{0}$ then $\langle a\rangle,\langle b\rangle,\langle a b\rangle$ are $a 11$ maximal subgroups of $Y_{0}$ and we have a contradiction.

CASE II. $M \cap M^{*} \neq\langle e\rangle$. Let $T=M \cap M *$ and observe $T \leq G$. Now consider $Y_{0} / T$. It is a p-group, $\frac{M}{T}, \frac{M^{*}}{T}$ are two maximal subgroups of $Y_{0} / T=\bar{Y}_{0}$ and $\bar{Y}_{0}$ does not have any other maximal subgroup besides $\frac{M}{T}=\bar{M}$ and $\bar{M}^{*}=\frac{M^{*}}{T}$. It follows therefore that $Y_{0} \in S \backslash S_{1}$ so that $\bar{Y}_{0}$ is cyclic and $Y_{0}=\langle x, T\rangle=\langle x\rangle$ since $T=\phi\left(Y_{0}\right)$ and we have a contradiction. Thus $S_{1}=\Phi$ and every element in $S$ is cyclic. But this implies every element $X$ in $S$ has exactly-one subgroup of index $p$ i.e. $X$ has got exactly one maximal subgroup. Hence $S$ must be empty also and therefore $G=P_{1} \times P_{2}$. Thus $P_{1}$ has exactly one maximal subgroup, $i=1,2$ and $P_{1}$ is therefore cyclic. Hence it follows that $G$ is cyclic also and the assertion in the lemma is proved.

LEMMA 2.4. A p-group with exactly two second maximal subgroups is necessarily a 2-group.

PROOF. Let $G$ be a counter example of the smallest possible order. Then every maximal subgroup $M$ of $G$ has exactly one maximal subgroup. (By Lemma 2.3 there is no $p-$ group with exactly two maximal subgroups.) Hence $M$ is cyclic and therefore by Theorem $H$ it now follows that $G$ is cyclic. This however, implies $G$ can have one second maximal subgroup which is a contradiction. Hence $G$ cannot exist and the assertion in the lemma follows.

LEMMA 2.5. A group $G$ with exactly two second maximal subgroups is necessarily supersolvable and $G$ is either a 2-group or else its order is divisible by two primes only.

PROOF. Every maximal subgroup of $G$ is elther of prime order or has one or two maximal subgroups. Therefore every maximal subgroup of $G$ is cyclic and consequently every Sylow subgroup of $G$ is cyclic. Hence $G$ is supersolvable and $G=P K, P \cap K=1$, $P \subseteq G$ where $P$ is the Sylow p-subgroup corresponding to the largest prime divisor of $|G|$ and $K$ is a p-complement. If $K=1$, then $G$ is a p-group and by Lemma 2.418 also a 2-group. Now suppose $|K| \neq 1$. To prove $|G|$ is divisible by two primes we may without 1088 of generality assume $G$ to be a counter example of the least possible order. If $K$ has one second maximal subgroup then by Theorem $1, K$ is a $q-g r o u p$ for some prime $q$ and if $K$ has two second maximal subgroups then $G$ being a counter example of least possible order, $K$ is either a 2-group or 1ts order is divisible by two primes. Suppose $K=R T$ where $R$ one $T$ are two sylow subgroups corresponding to the prime divisor $r$ and $t r>t$, 
of $|K|$. If $T_{1}<\cdot T, R_{1}<\cdot R$ then $R T{ }_{1}$ and $R_{1} T$ are maximal subgroups of $K$. (Note $R$ is cyclic and $R S K$ since $K$ is supersovable). Then PRT ${ }_{1}$ <. G. If $T_{2}<. T_{1}, P_{1}$ <. $P$, $R_{2}$ <. $R_{1}$ then $P R T_{2}, P_{1} R T_{1}, \quad P R_{2} T$ are three second maximal subgroups of $G$, a contradiction. (Any of $T_{2}, R_{2}, T_{1}, P_{1}$ could be $\langle e\rangle_{.}$) We thus need consider the case when $K$ has no second maximal subgroup. By Lemma $2.2,|K|$ is $p$ or $p^{2}$ or $q t, p, q, t$ are primes. We thus need only consider the case when $|K|=q t$. Let $G=P Q T$ where $Q$ and $T$ are Sylow subgroups of order $q$ and $t$ respectively and we may take $P, Q, T$ as a Sylow basis for $G$. IF $|P|=P$ then $P, Q, T$ are three second maximal subgroups of $G$. Now suppose $|\mathrm{P}|=\mathrm{p}^{\mathrm{n}}, \mathrm{n}>2$ and let $\mathrm{P}_{1}<$. $\mathrm{P}$ and $\mathrm{P}_{2}<$. $\mathrm{P}_{1}$. Then $\mathrm{P}_{2} \mathrm{QT}, \mathrm{P}_{1} \mathrm{Q}, \mathrm{P}_{1} \mathrm{~T}$ are three second maximal subgroups. Thus this case cannot exist and therefore there is no minimal counter example and the assertion in the theorem is proved.

We end our investigation of group structures through second maximal subgroup by proving the following proposition.

PROPOSITION 2.1. A group $G$ with exactly three second maximal subgroups is solvable.

PROOF. Evidently each maximal subgroup $M$ of $G$ contains one, two or three maximal subgroups. In the first two cases, $M$ is necessarily supersolvable. If $M$ has three maximal subgroups then each of these three subgroups is normal and therefore $M$ is nilpotent. For, if any of these maximal subgroups is not normal then it being its own normalizer it follows that all (three) maximal subgroups of $M$ are conjugate and therefore have the same index, which is impossible. Hence $G$ is solvable.

The next two propositions describe the structures of groups which have respectively one or two third maximal subgroups.

PROPOSITION 2.2. Let $G$ be a group with exactly one third maximal subgroup. Then all the sylow subgroups corresponding to odd primes are cyclic and a sylow 2-subgroup is either cyclic or else has a cyclic subgroup of index 2 .

PROOF. Evidently every second maximal subgroup is cyclic. If $P$ is a Sylow $p-$ subgroup of $G$ then since it is contained in some maximal subgroup of $G$, it follows by Theorem $H$ that $P$ has the desired property as claimed.

PROPOSITION 2.3. Let $G$ be a group with exactly two-thirds maximal subgroups. Then all the Sylow subgroups of $G$ are cyclic and $G$ is supersolvable.

PROOF. Follows immediately from Lemma 2.3 .

\section{REFERENCES}

1. HUPPERT, B., Endliche Gruppen I, Springer-Verlag, New York, 1967.

2. HUPPERT, B., Normal Teller and Maximale Untergruppen Endlicher Gruppen, Math. Z. 60 (1954), 409-434.

3. JANKO, A., Finite Groups with Invariant Fourth Maximal Subgroups, Math. Z. 82, (1963), 82-89.

4. WEINSTEIN, M., (Ed)., Between Nilpotent and Solvable, Polygonal Publishing House, NJ, USA.

5. BUCKLEY, J., Finite Groups whose Minimal Subgroups are Normal, Math. Z. 116 (1970), 15-17. 


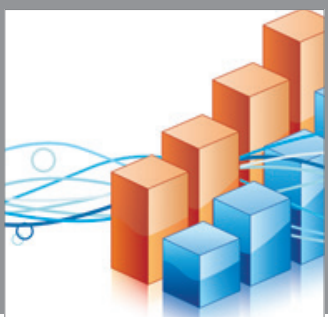

Advances in

Operations Research

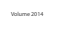

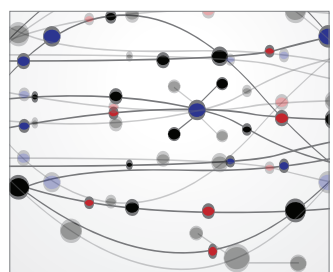

\section{The Scientific} World Journal
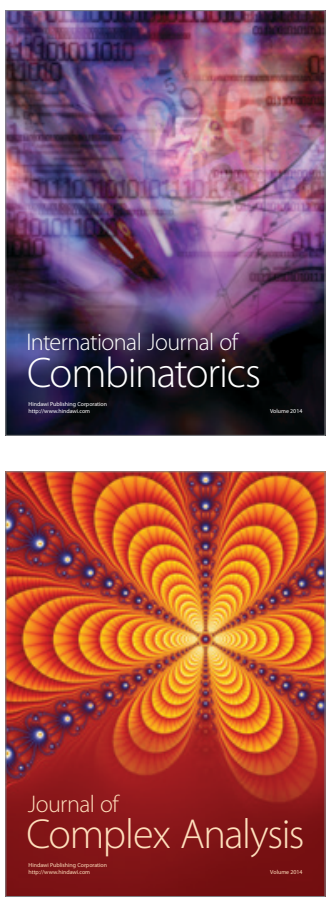

International Journal of

Mathematics and

Mathematical

Sciences
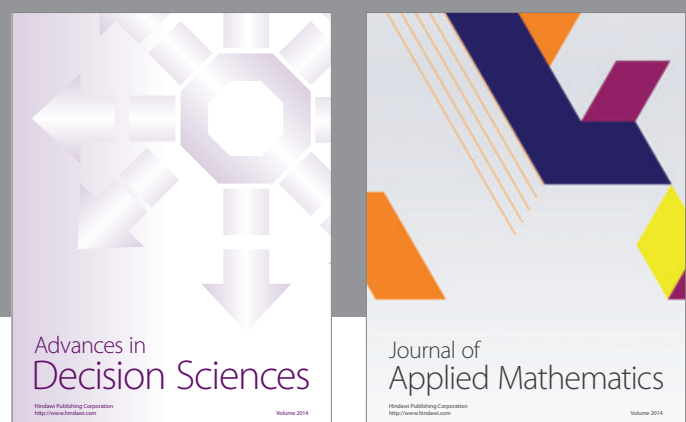

Journal of

Applied Mathematics
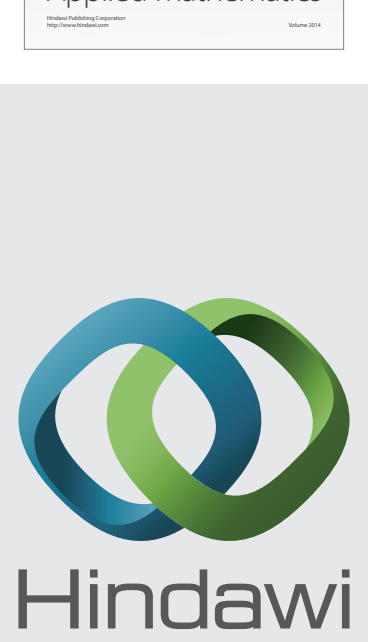

Submit your manuscripts at http://www.hindawi.com
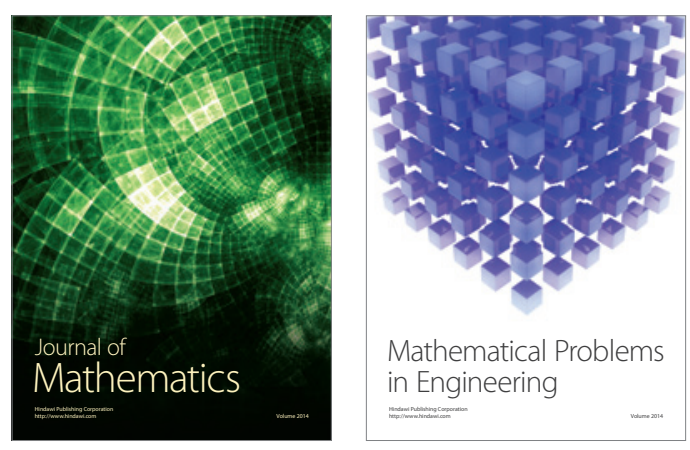

Mathematical Problems in Engineering
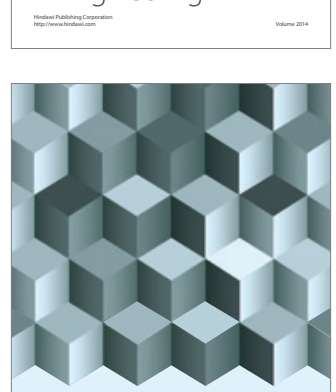

Journal of

Function Spaces
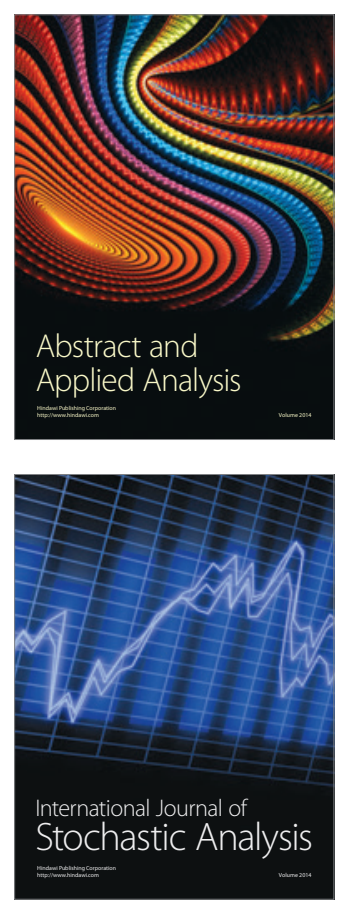

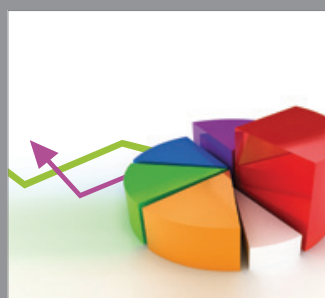

ournal of

Probability and Statistics

Promensencen
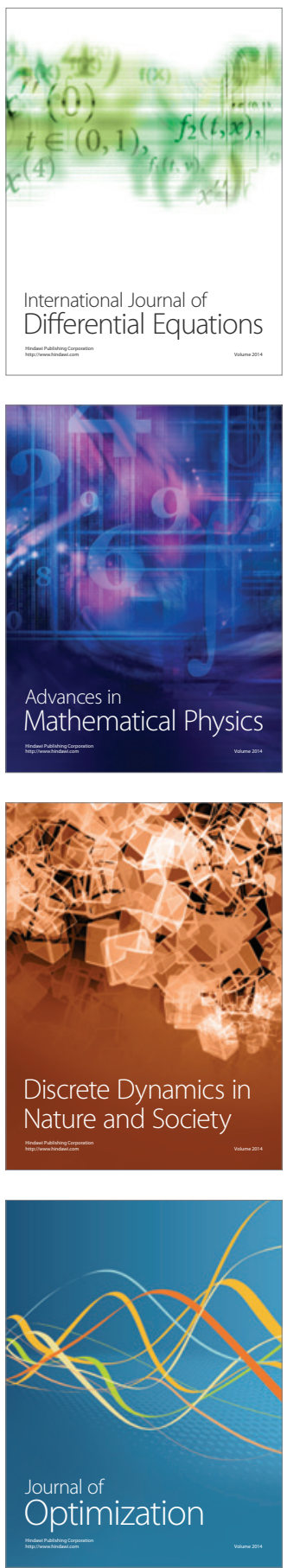